\title{
AN ELEMENTARY PROOF OF THE EXISTENCE OF ISOTHERMAL PARAMETERS ON A SURFACE
}

\author{
SHIING-SHEN CHERN
}

1. Introduction. Let

$$
\begin{aligned}
& d s^{2}=E(x, y) d x^{2}+2 F(x, y) d x d y+G(x, y) d y^{2}, \\
& E G-F^{2}>0, E>0,
\end{aligned}
$$

be a positive definite Riemann metric of two dimensions defined in a neighborhood of a surface with the local coordinates $x, y$. By isothermal parameters we mean local coordinates $u, v$ relative to which the metric takes the form

$$
d s^{2}=\lambda(u, v)\left(d u^{2}+d v^{2}\right), \quad \lambda(u, v)>0 .
$$

In order that isothermal parameters exist it is necessary to impose on the metric some regularity assumptions. In fact, it was shown recently by Hartman and Wintner ${ }^{1}$ that it is not sufficient to assume the functions $E, F, G$ to be continuous. So far the weakest conditions under which the isothermal parameters are known to exist were found by Korn and Lichtenstein. ${ }^{2}$ To formulate their theorem we recall that a function $f(x, y)$ in a domain $D$ of the $(x, y)$-plane is said to satisfy a Hölder condition of order $\lambda, 0<\lambda \leqq 1$, if the inequality

$$
\left|f(x, y)-f\left(x^{\prime}, y^{\prime}\right)\right|<C r^{\lambda}
$$

holds for any two points $(x, y),\left(x^{\prime}, y^{\prime}\right)$ of $D$, where $C$ is a constant and $r$ is the Euclidean distance between these two points. With this definition the theorem of Korn-Lichtenstein can be stated as follows:

Received by the editors September 29, 1954 and, in revised form, November 15, 1954.

1 P. Hartman and A. Wintner, On the existence of Riemannian manifolds which cannot carry non-constant analytic or harmonic functions in the small, Amer. J. Math. vol. 75 (1953) pp. 260-276. Also: S. Chern, P. Hartman, and A. Wintner, On isothermic coordinates, Comment-Math. Helv. vol. 28 (1954) pp. 301-309.

${ }^{2}$ A. Korn, Zwei Anwendungen der Methode der sukzessiven Annäherungen, Schwarz Abhandlungen pp. 215-229; L. Lichtenstein, Zur Theorie der konformen Abbildung. Konforme Abbildung nichtanalytischer, singularitätenfreier Flächenstïcke auf ebene Gebiete, Bull. Int. de l'Acad. Sci. Cracovie, ser. A (1916) pp. 192-217. Cf. also the paper of C. B. Morrey, On the solutions of quasi-linear elliptic partial differential equations, Trans. Amer. Math. Soc. vol. 43 (1938) pp. 126-166, in which the isothermal parameters are shown to exist in a generalized sense under weaker hypotheses.

Added in proof. Weaker conditions were recently found by Hartman and Wintner. Cf. their paper, On uniform Dini conditions in the theory of linear partial differential equations of elliptic type, Amer. J. Math. vol. 77 (1955) pp. 329-354. 
Suppose, in a domain $D$ of the $(x, y)$-plane, the functions $E, F, G$ satisfy a Hölder condition of order $\lambda, 0<\lambda<1$. Then every point of $D$ has a neighborhood whose local coordinates are isothermal parameters.

This theorem is rather useful in the global theory of surfaces, when analyticity assumptions are not desirable. We shall present here what seems to be an elementary and rather straightforward proof. Needless to say, the essential ideas of this proof are contained in the works of Korn and Lichtenstein. We believe, however, that some simplifications are achieved by the consistent use of the complex notation. ${ }^{3}$

We first observe that the isothermal parameters are invariant under conformal transformations, that is, multiplications of the Riemann metric (1) by a positive factor. Under a conformal transformation the angle between two vectors remains invariant. If we further assume that the coordinates $x, y$ form a positive system, that is, if we allow only those transformations of local coordinates for which the Jacobian is positive, the angle can be defined, together with its orientation. To express these relations analytically, it will be convenient to introduce complex-valued differential forms, that is, forms $\omega=\alpha+i \beta$, where $\alpha, \beta$ are real differential forms. We shall write $\bar{\omega}=\alpha-i \beta$.

Since the quadratic differential form in the right-hand side of (1) is positive definite, we can write

$$
d s^{2}=\theta_{1}^{2}+\theta_{2}^{2},
$$

where $\theta_{1}, \theta_{2}$ are real linear differential forms:

$$
\begin{aligned}
& \theta_{1}=a_{1} d x+b_{1} d y, \\
& \theta_{2}=a_{2} d x+b_{2} d y .
\end{aligned}
$$

Assuming that $a_{1} b_{2}-a_{2} b_{1}>0$, the forms $\theta_{1}, \theta_{2}$ are determined up to a proper orthogonal transformation, that is, one with determinant +1 . We put

$$
\phi=\theta_{1}+i \theta_{2}
$$

so that

$$
d s^{2}=\phi \bar{\phi} .
$$

The form $\phi$ is then determined up to a complex factor of absolute value 1. A conformal transformation of the Riemann metric is given,

${ }^{3}$ After this paper was submitted for publication, it has come to my attention that a similar proof was given by L. Bers in his mimeographed notes on Riemann surfaces, New York University, 1951-1952. 
in terms of the form $\phi$, by the multiplication of $\phi$ by an arbitrary nonzero complex factor. In the recent terminology we say that the complex linear differential form $\phi$, determined up to a nonzero complex factor, defines an almost complex structure. As discussed above, such a structure allows the introduction of the notion of oriented angle.

The determination of isothermal parameters $u, v$ is equivalent to that of a complex-valued function $w=u+i v$, such that

$$
d w=(1 / \rho) \phi .
$$

For we have then

$$
d s^{2}=\phi \bar{\phi}=|\rho|^{2} d w d \bar{w}=|\rho|^{2}\left(d u^{2}+d v^{2}\right) .
$$

Conversely, the isothermal parameters $u, v$ determine a function $w$ satisfying (8).

2. Preliminaries. We shall first make estimates of certain integrals, which will be needed in the proof.

Let $(\xi, \eta)$ be any point in the $(x, y)$-plane, and let

$$
r=+\left((x-\xi)^{2}+(y-\eta)^{2}\right)^{1 / 2} \text {. }
$$

If $g(r)$ is a function of class 1 , we have, by exterior differentiation,

$$
d\left\{g(\boldsymbol{r}) \frac{-(y-\eta) d x+(x-\xi) d y}{r^{2}}\right\}=\frac{g^{\prime}(\boldsymbol{r})}{r} d x \wedge d y .
$$

It follows by Stokes Theorem that, if $D$ is a domain bounded by a curve $C$ and if $(\xi, \eta) \notin D$, we have

$$
\iint_{D} \frac{g^{\prime}(r)}{r} d x d y=\int_{C} g(r) \frac{-(y-\eta) d x+(x-\xi) d y}{r^{2}} .
$$

This formula remains true, even if $(\xi, \eta) \in D$ (but not on $C$ ), provided that the integral in the left-hand side is convergent. The integral

$$
\nu=\frac{1}{2 \pi} \int_{C} \frac{-(y-\eta) d x+(x-\xi) d y}{r^{2}},
$$

is an integer and is usually called the order of the point $(\xi, \eta)$ relative to the curve $C$.

In particular, if $g(r)=r^{\lambda}, \lambda \neq 0$, formula (11) becomes

$$
\lambda \iint_{D} \frac{r^{\lambda} d x d y}{r^{2}}=\int_{C} r^{\lambda} \frac{-(y-\eta) d x+(x-\xi) d y}{r^{2}},
$$

and is true either when $(\xi, \eta) \notin D$ or when $\lambda>0$. It follows that, if the 
vector joining $(\xi, \eta)$ to a point $(x, y) \in C$ turns monotonely in the same sense, we have

$$
\begin{array}{ll}
\left|\iint_{D} \frac{r^{\lambda} d x d y}{r^{2}}\right| \leqq \frac{2 \pi}{\lambda} \Delta^{\lambda}, & \lambda>0, \\
\left|\iint_{D} \frac{r^{\lambda} d x d y}{r^{2}}\right| \leqq \frac{2 \pi}{|\lambda|} \delta^{\lambda}, & \lambda<0,
\end{array}
$$

where $\Delta$ and $\delta$ denote respectively an upper bound and a lower bound of the distance from $(\xi, \eta)$ to a point $(x, y) \in C$. We emphasize that formula (15) is valid only under the assumption $(\xi, \eta) \notin D$.

3. Main lemma. To simplify our formulas we shall use the complex coordinate $z=x+i y$ in the $(x, y)$-plane. If $f(x, y)$ is a complex-valued function of class 1 , we define $f_{z}, f_{z}$ by the equation

$$
d f=f_{z} d z+f_{\bar{z}} d \bar{z}=f_{z}(d x+i d y)+f_{\bar{z}}(d x-i d y) .
$$

They are therefore related to the ordinary partial derivatives $f_{x}, f_{y}$ by the equations

$$
f_{z}=\left(f_{x}-i f_{y}\right) / 2, \quad f_{\bar{z}}=\left(f_{x}+i f_{y}\right) / 2 .
$$

We shall write the function as $f(z, \bar{z})$, thus emphasizing the fact that it is in general not analytic in $z$.

On the other hand, we can define these operators $f_{z}, f_{\bar{z}}$ directly, without using the partial derivatives $f_{x}, f_{y}$. For instance, we can adopt the definitions:

$$
\begin{aligned}
f_{z}= & \lim _{h \rightarrow 0}\{f(z+h / 2, \bar{z}+h / 2)-f(z, \bar{z}) \\
& +i f(z-h i / 2, \bar{z}+h i / 2)-i f(z, \bar{z})\} / h, \\
f_{z}= & \lim _{h \rightarrow 0}\{f(z+h / 2, \bar{z}+h / 2)-f(z, \bar{z}) \\
& +i f(z+h i / 2, \bar{z}-h i / 2)-i f(z, \bar{z})\} / h,
\end{aligned}
$$

which clearly give (17), when $f_{x}, f_{y}$ exist.

Lemma. Let $D$ be a disc of radius $R$ about the origin in the $(x, y$-) plane. Let $f(z, \bar{z})$ be a complex-valued continuous function in $D$, which satisfies the condition

$$
\left|f\left(\zeta_{1}, \bar{\zeta}_{1}\right)-f\left(\zeta_{2}, \bar{\zeta}_{2}\right)\right| \leqq B r_{12}^{\lambda}, \quad r_{12}=\left|\zeta_{1}-\zeta_{2}\right|,
$$

for any two points $\zeta_{1}, \zeta_{2} \in D$, where $\lambda$ and $B$ are constants, $0<\lambda<1$. Let the function $F(\zeta, \bar{\zeta})$ be defined by 
(20) $-2 \pi i F(\zeta, \bar{\zeta})=\iint_{D} \frac{f(z, \bar{z}) d \bar{z} d z}{z-\zeta}=2 i \iint_{D} \frac{f(z, \bar{z}) d x d y}{z-\zeta}, \quad \zeta \in D$.

Then: (1) $F_{\zeta}$ and $F_{\bar{\zeta}}$ exist, and $F_{\bar{\zeta}}=f(\zeta, \bar{\zeta}) ;(2)$ If $|f(z, \bar{z})| \leqq A, z \in D$, the following inequalities are valid:

$$
\begin{aligned}
|F(\zeta, \bar{\zeta})| & \leqq 4 R A, \\
\left|F_{\zeta}(\zeta, \bar{\zeta})\right| & \leqq\left(2^{\lambda+1} / \lambda\right) R^{\lambda} B, \\
\left|F\left(\zeta_{1}, \bar{\zeta}_{1}\right)-F\left(\zeta_{2}, \bar{\zeta}_{2}\right)\right| & \leqq 2\left(A+\left(2^{\lambda+1} / \lambda\right) R^{\lambda} B\right) r_{12}, \\
\left|F_{\zeta}\left(\zeta_{1}, \bar{\zeta}_{1}\right)-F_{\zeta}\left(\zeta_{2}, \bar{\zeta}_{2}\right)\right| & \leqq \mu(\lambda) B r_{12}^{\lambda}
\end{aligned}
$$

where $\mu(\lambda)>0$ is independent of $\zeta_{1}, \zeta_{2}$.

To prove the lemma we write, according to the second equation of (18),

$$
\begin{aligned}
-2 \pi i F_{\bar{\zeta}}= & \lim _{h \rightarrow 0} \frac{1}{2} \iint_{D}(f(z, \bar{z})-f(\zeta, \bar{\zeta}))\left\{\frac{1}{(z-\zeta)(z-\zeta-h / 2)}\right. \\
& \left.-\frac{1}{(z-\zeta)(z-\zeta-h i / 2)}\right\} d \bar{z} d z+f(\zeta, \bar{\zeta}) \frac{\partial}{\partial \bar{\zeta}} \iint_{D} \frac{d \bar{z} d z}{z-\zeta} .
\end{aligned}
$$

Computation gives

$$
\iint_{D} \frac{d \bar{z} d z}{z-\zeta}=-2 \pi i \bar{\zeta}
$$

From these it follows that $F_{\bar{\zeta}}=f(\zeta, \bar{\zeta})$. Similarly, we prove that $F_{\zeta}$ exists and is given by

$$
-2 \pi i F_{\zeta}=\iint_{D} \frac{f(z, \bar{z})-f(\zeta, \bar{\zeta})}{(z-\zeta)^{2}} d \bar{z} d z .
$$

From (20) we have, by using (14) with $\lambda=1$,

$$
2 \pi|F| \leqq 2 A \iint_{D} \frac{d x d y}{r} \leqq 8 \pi A R,
$$

which gives (21). Similarly, from (26), we have

$$
2 \pi\left|F_{\zeta}\right| \leqq 2 B \iint_{D} \frac{r^{\lambda}}{r^{2}} d x d y \leqq \frac{2^{\lambda+2}}{\lambda} \pi B R^{\lambda},
$$

which gives (22).

Inequality (23) is trivial. To prove (24) let $D_{0}$ be the intersection 
of $D$ with a circular disc of radius $2 r_{12}=2\left|\zeta_{1}-\zeta_{2}\right|$ about $\zeta_{2}$. We can write

$-2 \pi i\left\{F_{\zeta}\left(\zeta_{1}, \bar{\zeta}_{1}\right)-F_{\zeta}\left(\zeta_{2}, \bar{\zeta}_{2}\right)\right\}$

$$
\begin{aligned}
=\iint_{D_{0}}\{f(z, \bar{z})- & \left.f\left(\zeta_{1}, \bar{\zeta}_{1}\right)\right\} \frac{1}{\left(z-\zeta_{1}\right)^{2}} d \bar{z} d z \\
& -\iint_{D_{0}}\left\{f(z, \bar{z})-f\left(\zeta_{2}, \bar{\zeta}_{2}\right)\right\} \frac{1}{\left(z-\zeta_{2}\right)^{2}} d \bar{z} d z \\
& +\iint_{D-D_{0}}\left\{f(z, \bar{z})-f\left(\zeta_{1}, \bar{\zeta}_{1}\right)\right\} \frac{1}{\left(z-\zeta_{1}\right)^{2}} d \bar{z} d z \\
& -\iint_{D-D_{0}}\left\{f(z, \bar{z})-f\left(\zeta_{1}, \bar{\zeta}_{2}\right)\right\} \frac{1}{\left(z-\zeta_{2}\right)^{2}} d \bar{z} d z \\
& +\left\{f\left(\zeta_{2}, \bar{\zeta}_{2}\right)-f\left(\zeta_{1}, \bar{\zeta}_{1}\right)\right\} \frac{\partial}{\partial \zeta_{2}} \iint_{D-D_{0}} \frac{d \bar{z} d z}{z-\zeta_{2}}
\end{aligned}
$$

We observe that in this sum only the first two integrals are improper integrals, while the last three are proper integrals. To estimate their absolute values we first have

$$
\begin{aligned}
& \left|\int_{D_{0}}\left\{f(z, \bar{z})-f\left(\zeta_{1}, \bar{\zeta}_{1}\right)\right\} \frac{1}{\left(z-\zeta_{1}\right)^{2}} d \bar{z} d z\right| \\
& \leqq 2 B \iint_{D_{0}} \frac{d x d y}{\left|z-\zeta_{1}\right|^{2-\lambda}} \leqq \frac{4 \pi B}{\lambda}\left(3 r_{12}\right)^{\lambda} \\
& \left|\int_{D_{0}}\left\{f(z, \bar{z})-f\left(\zeta_{2}, \bar{\zeta}_{2}\right)\right\} \frac{1}{\left(z-\zeta_{2}\right)^{2}} d \bar{z} d z\right| \\
& \leqq 2 B \iint_{D_{0}} \frac{d x d y}{\left|z-\zeta_{2}\right|^{2-\lambda}} \leqq \frac{4 \pi B}{\lambda}\left(2 r_{12}\right)^{\lambda}
\end{aligned}
$$

The sum of the third and fourth integrals in (27) is equal to

$$
\begin{aligned}
& \iint_{D-D_{0}}\left\{f(z, \bar{z})-f\left(\zeta_{1}, \bar{\zeta}_{1}\right)\right\} \int_{\zeta_{2}}^{\zeta_{1}} d \frac{1}{(z-\zeta)^{2}} d \bar{z} d z \\
& \quad=4 i \iint_{D-D_{0}} \int_{\zeta_{2}}^{\zeta_{1}}\left\{f(z, \bar{z})-f(\zeta, \bar{\zeta})+f(\zeta, \bar{\zeta})-f\left(\zeta_{1}, \zeta_{1}\right)\right\} \frac{1}{(z-\zeta)^{3}} d \zeta d x d y,
\end{aligned}
$$


where $\zeta$ is a point on the segment $\zeta_{1} \zeta_{2}$. It follows that its absolute value is

$$
\leqq 4 r_{12}\left(F_{1}+F_{2}\right) \text {, }
$$

where $F_{1}$ and $F_{2}$ are respectively upper bounds of the integrals

$$
\begin{gathered}
\left|\int_{D-D_{0}} \frac{f(z, \bar{z})-f(\zeta, \bar{\zeta})}{(z-\zeta)^{3}} d x d y\right|, \\
\left|\int_{D-D_{0}}\left\{f(\zeta, \bar{\zeta})-f\left(\zeta_{1}, \bar{\zeta}_{1}\right)\right\} \frac{d x d y}{(z-\zeta)^{3}}\right|
\end{gathered}
$$

along the segment $\zeta_{1} \zeta_{2}$. Using (15), we have

$$
\begin{aligned}
\left|\int_{D-D_{0}} \frac{f(z, \bar{z})-f(\zeta, \bar{\zeta})}{(z-\zeta)^{3}} d x d y\right| & \leqq B \int_{D-D_{0}} \frac{r^{\lambda-1} d x d y}{r^{2}} \\
& \leqq \frac{4 \pi B}{1-\lambda} \frac{1}{r_{12}^{1-\lambda}}, \\
\left|\int_{D-D_{0}} \frac{f(\zeta, \bar{\zeta})-f\left(\zeta_{1}, \bar{\zeta}_{1}\right)}{(z-\zeta)^{3}} d x d y\right| & \leqq B r_{12}^{\lambda} \frac{4 \pi}{r_{12}} .
\end{aligned}
$$

The right-hand members of these inequalities can therefore be taken as $F_{1}$ and $F_{2}$ respectively.

Finally, we have, by (25),

$$
\iint_{D-D_{0}} \frac{d \bar{z} d z}{z-\zeta_{2}}=\iint_{D} \frac{d \bar{z} d z}{z-\zeta_{2}}-\iint_{D_{0}} \frac{d \bar{z} d z}{z-\zeta_{2}}=-2 \pi i \xi_{2}
$$

the integral over $D_{0}$ being equal to zero. It follows that the last term of (27) is zero.

Summing up these estimates, we get

$$
\begin{aligned}
2 \pi \mid F_{\zeta}\left(\zeta_{1}, \bar{\zeta}_{1}\right)- & F_{\zeta}\left(\zeta_{2}, \bar{\zeta}_{2}\right) \mid \\
& \leqq \frac{4 \pi B}{\lambda}\left(3 r_{12}\right)^{\lambda}+\frac{4 \pi B}{\lambda}\left(2 r_{12}\right)^{\lambda}+4\left(\frac{4 \pi B}{1-\lambda}+4 \pi B\right) r_{12}^{\lambda} .
\end{aligned}
$$

This gives (24), if we set

$$
\mu(\lambda)=\frac{2}{\lambda}\left(3^{\lambda}+2^{\lambda}\right)+8 \frac{2-\lambda}{1-\lambda} .
$$

Thus the proof of the lemma is complete. 
4. An existence and uniqueness theorem on an integro-differential equation; application to isothermal parameters.

THEOREM. In the domain $D$ of the complex $z$-plane defined by $|z| \leqq R$ ( = positive constant) let

$$
Z w=a(z, \bar{z}) w_{\bar{z}}+b(z, \bar{z}) w_{z}
$$

be a differential operator, whose coefficients $a(z, \bar{z}), b(z, \bar{z})$ satisfy a Hölder condition of order $\lambda, 0<\lambda<1$, and vanish at $z=0$. Let $\alpha(z, \bar{z})$ be a function in $D$, satisfying a Hölder condition of the same order $\lambda$. Then the equation

$$
2 \pi i w(\zeta, \bar{\zeta})+\iint_{D} \frac{(Z w+\alpha w)(z, \bar{z})}{z-\zeta} d \bar{z} d z=\sigma(\zeta) \quad \zeta \in D,
$$

where $\sigma(\zeta)$ is a complex analytic function, with $\sigma(0)=0$, has exactly one solution $w(z, \bar{z})$, provided that $R$ is sufficiently small.

Before proceeding to the proof of this theorem, we shall make some further estimates of integrals, based on the lemma of the last section. The hypothesis of the theorem implies the existence of a number $M$ large enough to fulfill the following inequalities:

$$
\begin{gathered}
1<M, \quad|\alpha(\zeta, \bar{\zeta})| \leqq M, \quad|\sigma(\zeta)| \leqq M \\
\left|h\left(\zeta_{1}, \bar{\zeta}_{1}\right)-h\left(\zeta_{2}, \bar{\zeta}_{2}\right)\right| \leqq M r_{12}^{\lambda} \\
\left|(Z+\alpha) \sigma\left(\zeta_{1}\right)-(Z+\alpha) \sigma\left(\zeta_{2}\right)\right| \leqq \frac{M^{2}}{2^{\lambda}} r_{12}^{\lambda}, \quad r_{12}=\left|\zeta_{1}-\zeta_{2}\right|,
\end{gathered}
$$

where $\zeta, \zeta_{1}, \zeta_{2}$ are any three points of $D$ and where $h(z, \bar{z})$ stands for each of the functions $a(z, \bar{z}), b(z, \bar{z}), \alpha(z, \bar{z}), \sigma(z)$. Since $a, b,(Z+\alpha) \sigma$ all vanish at 0 , it follows from the corresponding Hölder inequalities that

$$
\begin{gathered}
|a(\zeta, \bar{\zeta})| \leqq M|\zeta|^{\lambda} \leqq M R^{\lambda}, \quad|b(\zeta, \bar{\zeta})| \leqq M R^{\lambda} \\
|(Z+\alpha) \sigma(\zeta)| \leqq M^{2} R^{\lambda}
\end{gathered}
$$

Consider now the function $F(\zeta, \bar{\zeta})$ defined in (20). Using the notation of the lemma of $\S 3$, we have

$$
\begin{aligned}
\mid(Z & +\alpha) F(\zeta, \bar{\zeta}) \mid \\
& =\left|a(\zeta, \bar{\zeta}) F_{\bar{\zeta}}+b(\zeta, \bar{\zeta}) F_{\zeta}+\alpha(\zeta, \bar{\zeta}) F\right| \\
& \leqq M R^{\lambda}\left(A+\left(2^{\lambda+1} / \lambda\right) R^{\lambda} B\right)+4 M R A \\
& =M R^{\lambda}\left\{\left(1+4 R^{1-\lambda}\right) A+\left(2^{\lambda+1} / \lambda\right) R^{\lambda} B\right\}
\end{aligned}
$$




$$
\begin{aligned}
&\left|(Z+\alpha) F\left(\zeta_{1}, \bar{\zeta}_{1}\right)-(Z+\alpha) F\left(\zeta_{2}, \bar{\zeta}_{2}\right)\right| \\
&=\mid a\left(\zeta_{1}, \bar{\zeta}_{1}\right) f\left(\zeta_{1}, \bar{\zeta}_{1}\right)+b\left(\zeta_{1}, \bar{\zeta}_{1}\right) F_{\zeta}\left(\zeta_{1}, \bar{\zeta}_{1}\right)+\alpha\left(\zeta_{1}, \bar{\zeta}_{1}\right) F\left(\zeta_{1}, \bar{\zeta}_{1}\right) \\
&-a\left(\zeta_{2}, \bar{\zeta}_{2}\right) f\left(\zeta_{2}, \bar{\zeta}_{2}\right)-b\left(\zeta_{2}, \bar{\zeta}_{2}\right) F_{\zeta}\left(\zeta_{2}, \bar{\zeta}_{2}\right)-\alpha\left(\zeta_{2}, \bar{\zeta}_{2}\right) F\left(\zeta_{2}, \bar{\zeta}_{2}\right) \mid \\
& \leqq M r_{12}^{\lambda}\left\{A+\left(2^{\lambda+1} / \lambda\right) R^{\lambda} B+4 R A\right\}+M R^{\lambda}(B+\mu(\lambda) B) r_{12}^{\lambda} \\
&+M\left(A+\left(2^{\lambda+1} / \lambda\right) R^{\lambda} B\right) \cdot 2 r_{12} \\
& M_{12}^{\lambda}\left\{A+g_{1}(R) A+g_{2}(R) B\right\},
\end{aligned}
$$

where

$$
\begin{aligned}
& g_{1}(R)=4 R+2^{2-\lambda} R^{1-\lambda}, \\
& g_{2}(R)=\left\{\left(2^{\lambda+1} / \lambda\right)+\mu(\lambda)+1\right\} R^{\lambda}+(8 / \lambda) R
\end{aligned}
$$

are functions of $R$, which tend to zero with $R$.

Having the above inequalities, we shall prove the existence of a solution of (30) by successive approximations. For reasons which will be clear later we put the following restrictions on $R$ :

$$
4 R^{\lambda} \leqq 1, \quad 2^{2-\lambda} \frac{\lambda+2}{\lambda} R^{1-\lambda} \leqq 1,
$$

and we choose a constant $c$, depending only on $\lambda$, such that

$$
\begin{array}{r}
\frac{\lambda+2}{\lambda}+\frac{\lambda}{\lambda+2} 2^{\lambda} \leqq c, \\
1+\mu(\lambda)+\frac{3 \lambda+2}{\lambda} 2^{\lambda} \leqq c .
\end{array}
$$

We now define

$$
\begin{aligned}
& 2 \pi i w_{0}(\zeta, \bar{\zeta})=\sigma(\zeta) \\
& 2 \pi i w_{n+1}(\zeta, \bar{\zeta})=-\iint_{D} \frac{\left(Z w_{n}+\alpha w_{n}\right)(z, \bar{z})}{z-\zeta} d \bar{z} d z, \quad n=0,1, \cdots
\end{aligned}
$$

For these functions we shall prove the inequalities:

$$
\text { (44) }\left|(Z+\alpha) w_{n}\left(\zeta_{1}, \bar{\zeta}_{1}\right)-(Z+\alpha) w_{n}\left(\zeta_{2}, \bar{\zeta}_{2}\right)\right| \leqq\left(c M^{2} / 2^{\lambda}\right)\left(c M R^{\lambda}\right)^{n} r_{12}^{\lambda} \text {. }
$$

In particular, the last one implies that the function under the integral 
sign in (40) satisfies a Hölder condition, thus allowing the definition of the next integral.

The inequalities (41)-(44) will be proved by induction on $n$. For $n=0,(41)$ is a consequence of the third inequality of (31), (42) follows from the third inequality of (34), since $c>1$, (43) follows from (32), and (44) from (33).

We now suppose that (41)-(44) are true and proceed to prove the corresponding inequalities for $n+1$. By (21), (23), and the induction hypothesis, we have

$$
\begin{aligned}
& \left|w_{n+1}(\zeta, \bar{\zeta})\right| \leqq 4 R \cdot M\left(c M R^{\lambda}\right)^{n+1} \leqq M\left(c M R^{\lambda}\right)^{n+1} \\
& \left|w_{n+1}\left(\zeta_{1}, \bar{\zeta}_{1}\right)-w_{n+1}\left(\zeta_{2}, \bar{\zeta}_{2}\right)\right| \\
& \leqq \\
& \quad 2 r_{12}\left\{M\left(c M R^{\lambda}\right)^{n+1}+\left(2^{\lambda+1} / \lambda\right) R^{\lambda}\left(c M^{2} / 2^{\lambda}\right)\left(c M R^{\lambda}\right)^{n}\right\} \\
& =M\left(c M R^{\lambda}\right)^{n+1} r_{12}^{\lambda}(1+2 / \lambda) \cdot 2 r_{12}^{1-\lambda} .
\end{aligned}
$$

The last relation gives (43) (for the index $n+1$ ), on account of the second inequality of (38).

Similarly, we get from (35) and the induction hypothesis,

$$
\begin{aligned}
\left|(Z+\alpha) w_{n+1}(\zeta, \bar{\zeta})\right| \leqq & M R^{\lambda}\left\{\left(1+4 R^{1-\lambda}\right) c M^{2} R^{\lambda}\right. \\
& \left.+\left(2^{\lambda+1} / \lambda\right) R^{\lambda}\left(c M^{2} / 2^{\lambda}\right)\right\}\left(c M R^{\lambda}\right)^{n} \\
= & M^{2} R^{\lambda}\left(c M R^{\lambda}\right)^{n+1}\left(1+4 R^{1-\lambda}+2 / \lambda\right) \\
\leqq & M\left(c M R^{\lambda}\right)^{n+2},
\end{aligned}
$$

on using the first inequality of (39). By (36), we have

$$
\begin{aligned}
&\left|(Z+\alpha) w_{n+1}\left(\zeta_{1}, \bar{\zeta}_{1}\right)-(Z+\alpha) w_{n+1}\left(\zeta_{2}, \bar{\zeta}_{2}\right)\right| \\
& \leqq M r_{12}^{\lambda} \cdot M\left(c M R^{\lambda}\right)^{n}\left\{\left(1+g_{1}(R)\right) c M R^{\lambda}+g_{2}(R) c M / 2^{\lambda}\right\} \\
&=M^{2} r_{12}^{\lambda}\left(c M R^{\lambda}\right)^{n+1}\left\{1+g_{1}(R)+\left(1 /(2 R)^{\lambda}\right) g_{2}(R)\right\} .
\end{aligned}
$$

This gives (44) for $n+1$, if

$$
1+g_{1}(R)+\left(1 /(2 R)^{\lambda}\right) g_{2}(R) \leqq c / 2^{\lambda} \text {. }
$$

But the latter follows from the second inequality of (39). Thus our induction is complete.

It follows that the series

$$
\sum_{n=0}^{\infty} w_{n}(z, \bar{z})
$$

converges absolutely and uniformly in $D$ if $C M R^{\lambda}<1$, and defines a function $w(z, \bar{z})$, which satisfies (30). 
To prove the uniqueness of the solution when $R$ is sufficiently small, let $w^{\prime}(z, \bar{z})$ be another solution of $(30)$ such that $(Z+\alpha) w^{\prime}(z, \bar{z})$ satisfies a Hölder condition of order $\lambda$. Then the function

$$
\tilde{w}(z, \bar{z})=w(z, \bar{z})-w^{\prime}(z, \bar{z})
$$

satisfies the equation

$$
-2 \pi i \tilde{w}(\zeta, \bar{\zeta})=\iint_{D} \frac{(Z+\alpha) \tilde{w}(z, \bar{z})}{z-\zeta} d \bar{z} d z
$$

Let $A$ and $B$ be respectively the least upper bounds of

$$
|(Z+\alpha) \tilde{w}(\zeta, \bar{\zeta})|, \zeta \in D
$$

and

$$
\left|(Z+\alpha) \tilde{w}\left(\zeta_{1}, \bar{\zeta}_{1}\right)-(Z+\alpha) \tilde{w}\left(\zeta_{2}, \bar{\zeta}_{2}\right)\right| / r_{12}^{\lambda}, \quad \zeta_{1}, \zeta_{2} \in D, \zeta_{1} \neq \zeta_{2} .
$$

From (35) and (36) we get

$$
\begin{aligned}
& A \leqq M R^{\lambda}\left\{\left(1+4 R^{1-\lambda}\right) A+\left(2^{\lambda+1} / \lambda\right) R^{\lambda} B\right\}, \\
& B \leqq M\left\{A+g_{1}(R) A+g_{2}(R) B\right\} .
\end{aligned}
$$

From these we easily conclude that, if $R$ is sufficiently small, $A=0$. The latter implies that $\tilde{w}(z, \bar{z})=0$. Thus the proof of our theorem is complete.

In order to derive from the above existence theorem the theorem of Korn-Lichtenstein we follow the notation of $\$ 1$. In a neighborhood of the point $z=x+i y=0$ we suppose the almost complex structure to be given by the complex-valued linear differential form

$$
\phi=(1-a(z, \bar{z})) d z+b(z, \bar{z}) d \bar{z},
$$

which is determined up to a nonzero factor. By a linear transformation on $x, y$ with constant coefficients and by multiplication of $\phi$ by a constant factor when necessary, we can suppose that $a(0,0)$ $=b(0,0)=0$. Equation (8) is equivalent to the equations

$$
w_{i}=b / \rho, \quad w_{z}=(1-a) / \rho .
$$

Elimination of $\rho$ gives

$$
(1-a) w_{z}-b w_{z}=0,
$$

or, if we make use of the operator $Z$ in (29),

$$
w_{i}=Z w .
$$

By the above existence theorem, the equation 


$$
2 \pi i w(\zeta, \bar{\zeta})+\iint_{D} \frac{Z w(z, \bar{z})}{z-\zeta} d \bar{z} d z=\sigma(\zeta) \quad \zeta \in D, \sigma(0)=0
$$

has a solution $w(z, \bar{z})$. If $\sigma(\zeta)$ is not a constant, say $\sigma(\zeta)=\zeta$, this solution has the property that $w_{\zeta}(0,0) \neq 0$. Since $Z w(z, \bar{z})$ satisfies a Hölder condition, it follows from our lemma in $\S 3$ that $w(z, \bar{z})$ satisfies (47). Thus we have proved the theorem of Korn-Lichtenstein.

REMARKs. 1. As L. Bers observed to me, the same method can be used to establish the existence of a local solution of the equation

$$
w_{\bar{z}}=a \bar{w}_{\bar{z}}+b w_{z}+\alpha w+\beta \bar{w}+\gamma,
$$

where $a, b, \alpha, \beta, \gamma$ satisfy a Hölder condition and where $|a|+|b|<1$.

2. It follows from our proof that the first partial derivatives of the isothermal parameters also satisfy a Hölder condition of order $\lambda$.

INSTITUTE For Advanced StUdy AND

University of Chicago 